\title{
Use of DWI-FLAIR Mismatch to Estimate the Onset Time in Wake-Up Strokes
}

\author{
Jinfeng Zhang $\mathbb{D}^{1,2}, \mathrm{Na} \mathrm{Ta}^{3}$, Meng $\mathrm{Fu}^{1,2}$, Fan Hua Tian $\mathbb{D}^{1,2}$, Jie Wang ${ }^{1,2}$, Tianyou Zhang ${ }^{1,2 *}$, \\ Baojun Wang ${ }^{1,2, *}$
}

'Department of Neurology, Baotou Central Hospital, Baotou, Inner Mongolia, People's Republic of China; ${ }^{2}$ Cerebrovascular Disease Research Institute of Inner Mongolia Autonomous Region, Baotou, Inner Mongolia, People's Republic of China; ${ }^{3}$ Practical Teaching Skills Center, Baotou Medical College, Inner Mongolia University of Science and Technology, Baotou, Inner Mongolia, People's Republic of China

*These authors contributed equally to this work

Correspondence: Tianyou Zhang; Baojun Wang, Department of Neurology, Baotou Central Hospital, Baotou, Inner Mongolia, People's Republic of China, Tel +86-472-6955I78; +86-472-6955346, Email jbwwbj@I26.com; 2533784284@qq.com

Purpose: To compare the MRI characteristics of patients with wake-up ischemic stroke (WUS) and with ischemic stroke with known onset time (clear-onset-time stroke, COS) to clarify the role of diffusion-weighted imaging-fluid-attenuated inversion recovery (DWIFLAIR) mismatch in estimating the onset time of WUS patients.

Patients and Methods: Two hundred patients with acute ischemic stroke were selected for complete brain MRI within six hours of symptom onset, including DWI and FLAIR sequences. The patients were divided into WUS $(\mathrm{n}=78)$ and COS $(n=122)$ groups, based on whether the time of onset was known. The general conditions and imaging characteristics were collected to compare the DWIFLAIR mismatch features between the two groups at different time intervals.

Results: There was no significant difference in the DWI-FLAIR mismatch on MRI within 2 hour after the first found abnormality between the two groups $(50.0 \%$ vs $71.8 \%, \mathrm{p}=0.180)$. With increasing time, the DWI-FLAIR mismatch decreased substantially in the WUS group, while a higher DWI-FLAIR mismatch presence persisted in the COS group within a four-hour interval from the onset of symptoms to the MRI. The DWI-FLAIR mismatch was significantly lower in the WUS group than in the COS group from symptom identification to MRI at 2-3 h, 3-4 h, and 4-5 h intervals ( $15 \%$ vs $60 \%, 10.5 \%$ vs $48 \%, 6.7 \%$ vs $45.4 \%$; $<<0.01)$.

Conclusion: Our results suggest that the presence of DWI-FLAIR mismatch within $2 \mathrm{~h}$ of the first found abnormality was not significantly different between WUS and COS. Therefore, Patients with WUS within 2 hours after the first detected abnormality may be suitable for intravenous thrombolysis.

Keywords: acute ischemic stroke, diffusion-weighted imaging, fluid-attenuated inversion recovery image, mismatch

\section{Introduction}

Ischemic strokes comprise $70 \%$ of all stroke cases; the most effective treatment in these cases is thrombolytic therapy within $4.5 \mathrm{~h}$ of onset. ${ }^{1,2}$ However, $25 \%$ of patients with ischemic stroke become aware of their symptoms upon awakening from sleep. International guidelines suggest to avoid intravenous thrombolysis in these patients due to insufficient evidence of safety and efficacy. ${ }^{3-5}$ A previous study found that most wake-up strokes (WUS) occur early in the morning, shortly before awakening; if these patients arrive at the hospital on time, the actual onset time may be estimated by a careful examination, and it could be possible to improve the prognosis with thrombolytic therapy. ${ }^{6}$ Some studies have proposed magnetic resonance imaging (MRI) diffusion-weighted imaging (DWI) and perfusion-weighted imaging (PWI) mismatches to identify WUS patients suitable for thrombolytic therapy. These imaging modalities may determine the presence of an ischemic semi-dark band and guide the use of thrombolytic therapy in WUS patients. ${ }^{7}$ The clinical application of PWI in WUS patients is limited by the short time window for treatment and the need for intravenous contrast injection, long imaging time, and post-processing of images. Recent studies have shown that patients with acute ischemic stroke presenting with DWI-positive lesions and no corresponding fluid-attenuated 
inversion recovery (FLAIR) lesions may benefit from timely thrombolytic therapy within $4.5 \mathrm{~h}$ of stroke onset. ${ }^{8}$ In this study, we compared MRI imaging characteristics of patients with WUS and stroke patients with known onset (clear-onset -time stroke, COS) to clarify the role of DWI-FLAIR mismatch in estimating the onset time of patients with WUS.

\section{Patients and Methods}

\section{Patients}

A total of 200 patients with acute ischemic stroke in the anterior circulation were selected retrospectively among the patients admitted to the Department of Neurology of Baotou Central Hospital from January 2018 to December 2020. The patients were divided into two groups, WUS $(n=78)$ and $\operatorname{COS}(n=122)$, based on whether the time of onset was clear. All patients met the diagnostic criteria for acute ischemic stroke diagnosis and 2018 treatment guidelines. ${ }^{9}$ Patients with a clear onset time and who underwent brain MRI within six hours were placed into the COS group. Patients who were asymptomatic before going to sleep and woke up with symptoms, or who were alone and could not report the exact onset time, who underwent head MRI within six hours from admission, were placed into the WUS group. The exclusion criteria were as follows: (1) MRI-confirmed presence of intracranial hemorrhage, tumor, infection, or cerebral infarct foci associated with the stroke symptoms; (2) Todd's palsy, hemiplegic migraine, or cervical spondylosis following focal epilepsy; (3) pacemakers, intravascular stents, or metal prostheses impeding MRI examinations.

General information was collected, including age, sex, time of last noted absence of symptoms, time of first evident abnormality, time of MRI, stroke risk factors, Trial of ORG 10172 in Acute Stroke Treatment (TOAST) classification of stroke, and evaluation of neurological deficits according to the National Institutes of Health Stroke Scale (NIHSS). The role of DWI-FLAIR mismatch in estimating the time of onset of ischemic stroke was assessed by comparing the DWI-FLAIR mismatch between the two groups based on the time interval between the first noted abnormality and MRI. Only in two cases, the MRI examination was completed within one hour of onset; thus, these patients were included with the 2 hour group for statistical purposes.

\section{MRI Data Acquisition Analysis}

The MRI was performed with a 1.5T MRI scanner (Siemens, Avanto, Germany), and the scan sequence was as follows: T1-weighted axial images with spin-echo sequence, repetition time: $450 \mathrm{~ms}$, echo time: $11 \mathrm{~ms}$; T2-weighted images with fast spin-echo sequence, repetition time: $5300 \mathrm{~ms}$, echo time: $110 \mathrm{~ms}$; T2-FLAIR sequence, repetition time: $9000 \mathrm{~ms}$, echo time: $109 \mathrm{~ms}$; DWI axial with single excitation spin-echo echo-planar sequence, repetition time: $3000 \mathrm{~ms}$, echo time: $89 \mathrm{~ms}$; while performing the apparent diffusion coefficient map, the diffusion sensitivity factor was $1000 \mathrm{~s} / \mathrm{mm}^{2}$. DWI positivity was defined as an area of high signal with $b=1000 \mathrm{~s} / \mathrm{mm}^{2}$, while the corresponding diffusion coefficient image showed a low signal; T2-FLAIR positivity was defined as the presence of an area of high signal in the region corresponding to the DWI-positive lesion. DWI-FLAIR mismatch was defined as a DWI-positive and T2-FLAIRnegative area; if two or more lesions were present simultaneously, all lesions were considered for the above criteria. Image interpretation was performed by two neuroimaging experts blinded to the clinical features of the patient; in case of disagreement, the final decision was based on the assessment by another senior neuroimaging expert.

\section{Statistical Analysis}

The statistical analysis was performed using SPSS 19.0 (IBM, Armonk, NY, USA); the $t$-test was used for comparison between groups for numerical data using $\bar{x} \pm \mathrm{s}$, which conformed to normally distributed data, and the Mann-Whitney $U$-test was used for group comparison for non-normally distributed data. The $\chi^{2}$ test was used to compare groups for categorical data, and logistic regression was used to analyze the factors affecting the DWI-FLAIR mismatch. A 2-tailed $\mathrm{P}<0.05$ was considered statistically significant. Divided into five groups according to time, we used Bonferroni's correction. The alpha value taken to be statistically significant is 0.05 divided by 5 equals 0.01 . 
Table I Comparison of Baseline Characteristics and Proportion of DWI-FLAIR Mismatch Between Patients with WUS and COS

\begin{tabular}{|c|c|c|c|}
\hline & WUS (78) & $\cos (122)$ & $\mathbf{P}$ \\
\hline Age, y & $69.5 \pm 7.3$ & $61.3 \pm 10.4$ & 0.044 \\
\hline Male, n (\%) & $42(62.6)$ & $90(67.7)$ & 0.376 \\
\hline NIHSS & $10.34 \pm 6.21$ & $6.25 \pm 6.67$ & 0.002 \\
\hline Hypertension, n (\%) & $45(67.1)$ & $93(69.9)$ & 0.655 \\
\hline Diabetes, n (\%) & $19(28.3)$ & $33(24.8)$ & 0.557 \\
\hline Hyperliperima, n (\%) & $24(35.8)$ & $62(46.6)$ & 0.108 \\
\hline Smoke, n (\%) & $21(3 \mid .3)$ & $43(32.3)$ & 0.905 \\
\hline Stroke history, n (\%) & $20(29.9)$ & $37(27.8)$ & 0.702 \\
\hline Atrial fibrillation, $n$ (\%) & $23(34.3)$ & $4 I(30.8)$ & 0.558 \\
\hline TOAST, n (\%) & & & 0.820 \\
\hline $\begin{array}{l}\text { Large artery } \\
\text { atherosclerosis }\end{array}$ & $25(37.3)$ & $55(4 I .4)$ & \\
\hline Cardioembolism & $26(38.8)$ & $46(34.6)$ & \\
\hline Small vessel occlusion & $8(11.9)$ & $16(12.0)$ & \\
\hline Other & $3(4.5)$ & $10(7.5)$ & \\
\hline Undetermined & $5(7.4)$ & $6(4.5)$ & \\
\hline LNT-MRI time, min & $553.2 \pm 486.2$ & $231.4 \pm 85.0$ & $<0.001$ \\
\hline FAT-MRI time, $\min$ & $211.3 \pm 72.1$ & $231.3 \pm 84.0$ & 0.353 \\
\hline DWI-FLAIR Mismatch, $n$ & & & \\
\hline 3h within MRI & $34(9)$ & $63(42)$ & $<0.001$ \\
\hline 4.5h within MRI & $61(13)$ & $97(59)$ & $<0.001$ \\
\hline
\end{tabular}

Abbreviations: WUS, wake-up ischemic stroke; COS, clear-onset time stroke; LNT, last known normal Time; FAT, first found abnormal time.

\section{Results}

\section{Comparison of Group Baseline Characteristics}

The WUS group included 78 patients, and the COS group 122. In the WUS group, 56 patients had symptoms after awakening, while 22 had no witnesses present at onset, suffered from aphasia, and could not report the exact onset time. Age and NIHSS score at admission were significantly higher in the WUS group than in the COS group $(\mathrm{p}=0.044$, $\mathrm{p}=0.002$, respectively), and the percentage of DWI-FLAIR mismatch on MRI within $3 \mathrm{~h}$ and $4.5 \mathrm{~h}$ after first found symptoms was significantly lower than in the COS group $(\mathrm{p}<0.001$, Table 1$)$.

\section{Group Comparison of DWI-FLAIR Mismatch}

In all patients, the DWI-FLAIR mismatch decreased gradually with increasing time from symptom onset to MRI examination. Twenty-nine cases $(63.0 \%)$ of DWI-FLAIR mismatch were found within $2 \mathrm{~h}$ from symptom onset $(7 / 14$ [50.0\%] in WUS and 22/32 [71.8\%] in COS), and the difference was not statistically significant $(p=0.180)$. However, with increasing time, the presence of DWI-FLAIR mismatch decreased substantially in the WUS group, while a higher number of DWI-FLAIR mismatches was still present in the COS group in the intervals from 2 to $5 \mathrm{~h}$. DWI-FLAIR mismatch in the WUS group was significantly lower than in the COS group in the $2-3 \mathrm{~h}, 3-4 \mathrm{~h}$, and $4-5 \mathrm{~h}$ intervals from symptom onset to MRI ( $\mathrm{p}=0.001, \mathrm{p}=0.005, \mathrm{p}=0.009$, respectively) $(\mathrm{P}<0.01$, Table 2$)$.

\section{Multifactorial Logistic Regression Analysis}

The NIHSS score at admission and the time from symptom onset to MRI examination between 2 and $6 \mathrm{~h}$ were prominently associated with DWI-FLAIR mismatch and were predictors of DWI-FLAIR mismatch. Although the p-value for the 2-3h outcome was located near the critical value $(\mathrm{p}=0.04)$ compared with less than $2 \mathrm{~h}$, its OR was smaller. The exposure was associated with a 0.394 -fold increased rate of the outcome (95\% confidence interval, $0.178-0.759)$ (Table 3 ). 
Table 2 Proportion of Patients with WUS and COS with DWI-FLAIR Mismatch According to the Interval Between FirstFound Abnormal and MRI Time

\begin{tabular}{|l|c|c|c|c|c|}
\hline & \multicolumn{2}{|c|}{ WUS (78) } & \multicolumn{2}{c|}{ COS (122) } & \multirow{2}{*}{ P } \\
\cline { 2 - 6 } & N & DWI-FLAIR Mismatch & N & DWI-FLAIR Mismatch & \\
\hline$\leq 2 \mathrm{~h}$ & 14 & $7(50.0)$ & 32 & $22(71.8)$ & 0.180 \\
$2-3 \mathrm{~h}$ & 20 & $3(15.0)$ & 30 & $18(60.0)$ & 0.001 \\
$3-4 \mathrm{~h}$ & 19 & $2(10.5)$ & 25 & $12(48.0)$ & 0.005 \\
$4-5 \mathrm{~h}$ & 15 & $1(6.7)$ & 22 & $10(45.4)$ & 0.009 \\
$5-6 \mathrm{~h}$ & 10 & $1(10.0)$ & 13 & $2(15.4)$ & 0.580 \\
\hline
\end{tabular}

Table 3 Multifactorial Logistic Regression Analysis

\begin{tabular}{|l|c|c|c|}
\hline & OR & $\mathbf{9 5 \%} \mathbf{~ C I}$ & $\mathbf{P}$ \\
\hline Age & $0.91 \mathrm{0}$ & $0.896 \sim 0.997$ & 0.540 \\
NIHSS & 0.935 & $0.896 \sim 0.997$ & $0.01 \mathrm{I}$ \\
FAT-MRI time & & & - \\
$\leq 2 \mathrm{~h}$ & 1.000 & - & 0.040 \\
2-3h & 0.394 & $0.178 \sim 0.759$ & 0.007 \\
$3-4 \mathrm{~h}$ & 0.317 & $0.137 \sim 0.742$ & 0.003 \\
4-5h & 0.179 & $0.063 \sim 0.530$ & $0.00 \mathrm{I}$ \\
5-6h & 0.067 & $0.018 \sim 0.270$ & \\
\hline
\end{tabular}

\section{Discussion}

DWI represents the diffusion of water molecules in the body, and it has been reported that patients with ischemic stroke can present DWI signal changes within minutes of onset. ${ }^{10-12}$ FLAIR is an inversion recovery sequence that highlights the signal contrast between the lesion and surrounding tissues by selecting a delay time (TI) close to the T1 value of the cerebrospinal fluid to produce a significant signal attenuation in the cerebrospinal fluid and tissues with similar T1 attenuation. Pathological studies $^{12}$ suggested that Intracellular edema can occur early after brain ischemia, the mechanism is that after the ischemia of brain tissue reaches the threshold of cell membrane pump failure, Cell membrane ion pump transport disorder, sodium, calcium ions and water in extracellular fluid enter cells, resulting in cytotoxic brain edema. At this time, the total water content in the ischemic area did not increase, but the ratio of water inside and outside the cells changed, so neither T2WI nor FLAIR showed abnormality. However, the ability of water molecules to diffuse and move in the cell is weaker than that outside the cell, resulting in high DWI signal in the lesion area. Thomalla et $\mathrm{a}^{13}$ found that ischemic stroke could be invisible on FLAIR images within $3 \mathrm{~h}$ of stroke onset and proposed the DWI-FLAIR mismatch model. Other researchers reported the mismatch as a marker to identify patients who are within $4.5 \mathrm{~h}$ of onset. Thomalla et $\mathrm{al}^{8}$ performed a multicenter observational study in acute ischemic stroke patients within $4.5 \mathrm{~h}$ of onset. It showed that DWI-FLAIR mismatch could identify patients within 4.5 $\mathrm{h}$ with a specificity of 0.78 and a PPV (positive predictive value) of 0.83 . These results were consistent with other studies. ${ }^{14,15}$ Early intravenous thrombolysis is the only treatment proven effective in acute ischemic stroke; however, previous studies on awakening stroke are scarce, resulting in this group of patients not receiving intravenous thrombolytic therapy after admission to the hospital. The application of the DWI-FLAIR mismatch model may help improve this situation. ${ }^{16-18}$

This study found that wake-up stroke has generally more severe clinical symptoms compared with acute ischemic stroke with a clear time of onset. The increased severity predicts a poorer long-term prognosis and a heavier burden on the patient, the family, and society, as seen in the results of previous studies. ${ }^{4,19}$ In this study, we found that DWI-FLAIR mismatch decreased gradually as the time from symptom onset to MRI increased, and DWI-FLAIR mismatches were more frequent in the COS group than in the WUS group within $4.5 \mathrm{~h}$ from the appearance of symptoms to MRI; in contrast, there was no significant difference between the two groups within $2 \mathrm{~h}$ from the first noted symptoms to MRI examination. The analysis based on different time intervals from the appearance of symptoms to MRI showed that the proportion of DWI-FLAIR 
mismatch in the WUS group decreased substantially when the interval from the first abnormality to MRI was longer than $2 \mathrm{~h}$; this trend was significantly different in the COS group. The presence of a high-signal lesion in FLAIR images indicates vasogenic edema of the brain parenchyma, and its high signal is a continuous change. Our study suggests that the DWIFLAIR mismatch continued to decrease with increasing time from onset, implying that more evident alterations in the FLAIR signal were detected on MRI after a longer time from onset. This study confirmed that DWI-FLAIR mismatch can be used as a helpful imaging tool to estimate the time of onset and could be used to guide the use of thrombolytic therapy in acute ischemic stroke patients when the time of onset cannot be determined. Previous studies showed that DWI-FLAIR mismatch decreased with increasing time from the onset at a rate of $8.1 \% / \mathrm{h},{ }^{8} 9.5 \% / \mathrm{h},{ }^{20}$ and $13.2 \% / \mathrm{h} .{ }^{13}$ Our study showed a similar rate of change in the COS group $(12 \% / \mathrm{h})$. The WUS group showed a $35 \%$ abrupt change between less than $2 \mathrm{~h}$ and 2-3 $\mathrm{h}$ from the onset. The comparison of the WUS and the COS group showed a decrease in the predictive validity of DWIFLAIR mismatch after more than $4.5 \mathrm{~h}$ from the time of first abnormality detection to MRI. The WUS and COS groups showed a reduced predictive validity of DWI-FLAIR mismatch depending on the time of first symptom detection; the WUS and COS groups showed a similar rate of DWI-FLAIR mismatch within a $2 \mathrm{~h}$ interval from the first found symptoms to the MRI. This similarity may be explained by the fact that the WUS likely occurred near the moment of awakening, and the symptoms were more severe than in COS patients; therefore, the patients arrived at the hospital promptly.

Our study has some noteworthy limitations. First, it was retrospective in nature and had a relatively small sample size. Furthermore, we focused on DWI-positive and FLAIR-negative lesions without further analysis of other possible small lesions or changes on the FLAIR images, which may have some impact on the patient's prognosis.

\section{Conclusion}

In summary, thrombolysis or revascularization beyond the $4.5 \mathrm{~h}$ time window is closely associated with intracranial hemorrhage and poor prognosis. ${ }^{21-23}$ It is important to choose an appropriate thrombolytic therapy for WUS. ${ }^{24-26}$ The last detected normal time is commonly used to determine if thrombolysis is appropriate; however, in patients with WUS, the time from the last-noted normal appearance to the time of consultation is longer and not representative. Our study indicates that the presence of DWI-FLAIR mismatch within $2 \mathrm{~h}$ of the first found abnormality was not significantly different between WUS and COS. Therefore, patients with WUS whose symptoms are first detected within $2 \mathrm{~h}$ may be good candidates for DWI-FLAIR mismatch based thrombolysis. We will further investigate the safety and efficacy of the thrombolytic therapy in patients selected by DWI-FLAIR mismatch in the future.

\section{Ethics Statement}

The studies involving human participants were reviewed and approved by Ethical Committee of Baotou Central Hospital. The patients/participants provided their written informed consent to participate in this study.

\section{Author Contributions}

All authors made a significant contribution to the work reported, whether that is in the conception, study design, execution, acquisition of data, analysis and interpretation, or in all these areas; took part in drafting, revising or critically reviewing the article; gave final approval of the version to be published; have agreed on the journal to which the article has been submitted; and agree to be accountable for all aspects of the work.

\section{Funding}

The authors have no funding to declare.

\section{Disclosure}

The authors declare that the research was conducted in the absence of any commercial or financial relationships that could be construed as a potential conflict of interest. 


\section{References}

1. National Institute of Neurological Disorders and Stroke rt-PA Stroke Study Group. Tissue plasminogen activator for acute ischemic stroke. $N$ Engl J Med. 1995;333(24):1581-1587. doi:10.1056/NEJM199512143332401

2. Hacke W, Kaste M, Bluhmki E, et al. Thrombolysis with alteplase 3 to 4.5 hours after acute ischemic stroke. $N$ Engl J Med. 2008;359 (13):1317-1329. doi:10.1056/NEJMoa0804656

3. Kang DW, Kwon JY, Kwon SU, Kim JS. Wake-up or unclear-onset strokes: are they waking up to the world of thrombolysis therapy? Int J Stroke. 2012;7(4):311-320. doi:10.1111/j.1747-4949.2012.00779.x

4. Mackey J, Kleindorfer D, Sucharew H, et al. Population-based study of wake-up strokes. Neurology. 2011;76(19):1662-1667. doi:10.1212/ WNL.0b013e318219fb30

5. Zhang YL, Zhang JF, Wang XX, Wang Y, Anderson CS, Wu YC. Wake-up stroke: imaging-based diagnosis and recanalization therapy. $J$ Neurol. 2021;268(11):4002-4012. doi:10.1007/s00415-020-10055-7

6. Rimmele DL, Thomalla G. Wake-up stroke: clinical characteristics, imaging findings, and treatment option - an update. Front Neurol. 2014;5:35. doi:10.3389/fneur.2014.00035

7. Warach S, Al-Rawi Y, Furlan AJ, et al. Refinement of the magnetic resonance diffusion-perfusion mismatch concept for thrombolytic patient selection: insights from the desmoteplase in acute stroke trials. Stroke. 2012;43(9):2313-2318. doi:10.1161/STROKEAHA.111.642348

8. Thomalla G, Cheng B, Ebinger M, et al. DWI-FLAIR mismatch for the identification of patients with acute ischaemic stroke within $4 \cdot 5 \mathrm{~h}$ of symptom onset (PRE-FLAIR): a multicentre observational study. Lancet Neurol. 2011;10(11):978-986. doi:10.1016/S1474-4422(11)70192-2

9. Powers WJ, Rabinstein AA, Ackerson T, et al. 2018 Guidelines for the early management of patients with acute ischemic stroke: a guideline for healthcare professionals from the American Heart Association/American Stroke Association. Stroke. 2018;49(3):e46-e110. doi:10.1161/ STR.0000000000000158

10. Madai VI, Galinovic I, Grittner U, et al. DWI intensity values predict FLAIR lesions in acute ischemic stroke. PLoS One. 2014 ;9(3):e92295. doi:10.1371/journal.pone.0092295

11. Chien D, Kwong KK, Gress DR, Buonanno FS, Buxton RB, Rosen BR. MR diffusion imaging of cerebral infarction in humans. Am J Neuroradiol. 1992;13(4):1097-1102.

12. Wouters A, Lemmens R, Dupont P, Thijs V. Wake-up stroke and stroke of unknown onset: a critical review. Front Neurol. 2014;5:153. doi:10.3389/ fneur.2014.00153

13. Thomalla G, Rossbach P, Rosenkranz M, et al. Negative fluid-attenuated inversion recovery imaging identifies acute ischemic stroke at 3 hours or less. Ann Neurol. 2009;65(6):724-732. doi:10.1002/ana.21651

14. Wei XE, Zhou J, Li WB, Zhao YW, Li MH, Li YH. MRI based thrombolysis for FLAIR-negative stroke patients within 4.5-6h after symptom onset. J Neurol Sci. 2017;372:421-427. doi:10.1016/j.jns.2016.11.010

15. Emeriau S, Serre I, Toubas O, Pombourcq F, Oppenheim C, Pierot L. Can diffusion-weighted imaging-fluid-attenuated inversion recovery mismatch (positive diffusion-weighted imaging/negative fluid-attenuated inversion recovery) at 3 Tesla identify patients with stroke at $<4.5$ hours? Stroke. 2013;44(6):1647-1651. doi:10.1161/STROKEAHA.113.001001

16. Kim BJ, Kim HJ, Lee DH, et al. Diffusion-weighted image and fluid-attenuated inversion recovery image mismatch: unclear-onset versus clear-onset stroke. Stroke. 2014;45(2):450-455. doi:10.1161/STROKEAHA.113.002830

17. Aoki J, Kimura K, Iguchi Y, et al. Intravenous thrombolysis based on diffusion-weighted imaging and fluid-attenuated inversion recovery mismatch in acute stroke patients with unknown onset time. Cerebrovasc Dis. 2011;31(5):435-441. doi:10.1159/000323850

18. Kang DW, Sohn SI, Hong KS, et al. Reperfusion therapy in unclear-onset stroke based on MRI evaluation (RESTORE): a prospective multicenter study. Stroke. 2012;43(12):3278-3283. doi:10.1161/STROKEAHA.112.675926

19. Bai Q, Zhao Z, Fu P, et al. Clinical outcomes of fast MRI-based thrombolysis in wake-up strokes compared to superacute ischemic strokes within 12 hours. Neurol Res. 2013;35(5):492-497. doi:10.1179/1743132813Y.0000000208

20. Ebinger M, Galinovic I, Rozanski M, Brunecker P, Endres M, Fiebach JB. Fluid-attenuated inversion recovery evolution within 12 hours from stroke onset: a reliable tissue clock? Stroke. 2010;41(2):250-255. doi:10.1161/STROKEAHA.109.568410

21. Jiménez-Conde J, Ois A, Rodríguez-Campello A, Gomis M, Roquer J. Does sleep protect against ischemic stroke? Less frequent ischemic strokes but more severe ones. $J$ Neurol. 2007;254(6):782-788. doi:10.1007/s00415-006-0438-y

22. Lees KR, Bluhmki E, von Kummer R, et al. Time to treatment with intravenous alteplase and outcome in stroke: an updated pooled analysis of ECASS, ATLANTIS, NINDS, and EPITHET trials. Lancet. 2010;375(9727):1695-1703. doi:10.1016/S0140-6736(10)60491-6

23. Nadeau JO, Fang J, Kapral MK, Silver FL, Hill MD; Registry of the Canadian Stroke Network. Outcome after stroke upon awakening. Can J Neurol Sci. 2005;32(2):232-236. doi:10.1017/s0317167100004029

24. Mishra NK, Albers GW, Davis SM, et al. Mismatch-based delayed thrombolysis: a meta-analysis. Stroke. 2010;41(1):e25-e33. doi:10.1161/ STROKEAHA.109.566869

25. Silva GS, Lima FO, Camargo EC, et al. Wake-up stroke: clinical and neuroimaging characteristics. Cerebrovasc Dis. 2010;29(4):336-342. doi:10.1159/000278929

26. Breuer L, Schellinger PD, Huttner HB, et al. Feasibility and safety of magnetic resonance imaging-based thrombolysis in patients with stroke on awakening: initial single-centre experience. Int $J$ Stroke. 2010;5(2):68-73. doi:10.1111/j.1747-4949.2010.00410.x 


\section{Publish your work in this journal}

Neuropsychiatric Disease and Treatment is an international, peer-reviewed journal of clinical therapeutics and pharmacology focusing on concise rapid reporting of clinical or pre-clinical studies on a range of neuropsychiatric and neurological disorders. This journal is indexed on PubMed Central, the 'PsycINFO' database and CAS, and is the official journal of The International Neuropsychiatric Association (INA). The manuscript management system is completely online and includes a very quick and fair peer-review system, which is all easy to use. Visit http://www.dovepress.com/testimonials.php to read real quotes from published authors. 